\title{
Identifying the Molecular Origin of Global Warming ${ }^{\dagger}$
}

\author{
Partha P. Bera ${ }^{1 \mathrm{a}}$, Joseph S. Francisco ${ }^{2}$, and Timothy J. Lee ${ }^{1 \mathrm{~b} *}$ \\ ${ }^{1}$ NASA Ames Research Center, Space Science and Astrobiology Division, \\ Moffett Field, CA, 94035 \\ ${ }^{2}$ Department of Chemistry and Department of Earth and Atmospheric Sciences \\ Purdue University
}

West Lafayette, Indiana 47907-1393

Corresponding Author E-mail: Timothy.J.Lee@,nasa.gov

Title Running Head: Identifying the Molecular Origin of Global Warming

Keywords: global warming, greenhouse effect, CFC, HFC, PFC, atmospheric change, climate change, GWP, global warming potential, ghg

\section{Abstract}

\footnotetext{
$\dagger$ Part of the "Russell Pitzer Festschrift"

${ }^{\text {a } M S 245-6 ~}$

${ }^{\mathrm{b}} \mathrm{MS} 245-1$
} 
We have investigated the physical characteristics of greenhouse gases (GHGs) to assess which properties are most important in determining the efficiency of a GHG. Chlorofluorcarbons (CFCs), hydrofluorocarbons (HFCs), perfluorocarbons (PFCs), nitrogen fluorides, and various other known atmospheric trace molecules have been included in this study. Compounds containing the halogens $\mathrm{F}$ or $\mathrm{Cl}$ have in common very polar $\mathrm{X}-\mathrm{F}$ or $\mathrm{X}-\mathrm{Cl}$ bonds, particularly the $\mathrm{X}-\mathrm{F}$ bonds. It is shown that as more $\mathrm{F}$ atoms bond to the same central atom, the bond dipoles become larger as a result of the central atom becoming more positive. This leads to a linear increase in the total or integrated XF bond dipole derivatives for the molecule, which leads to a non-linear (quadratic) increase in infrared (IR) intensity. Moreover, virtually all of the X-F bond stretches occur in the atmospheric IR window as opposed to $\mathrm{X}-\mathrm{H}$ stretches, which do not occur in the atmospheric window. It is concluded that molecules possessing several $\mathrm{F}$ atoms will always have a large radiative forcing parameter in the calculation of their global warming potential. Some of the implications for global warming and climate change are discussed. 


\section{Introduction}

Global climate change is regarded as one of the most significant scientific challenges to address in the coming decades. Temperature equilibrium in the Earth's atmosphere is maintained by a balanced absorption and emission of all the electromagnetic radiation reaching the surface of the Earth. Over the last few decades an increasing trend in the global surface temperature has been caused, at least in part, by an increased concentration of greenhouse gasses (GHG). Gases in the atmosphere play a vital role in maintaining this delicate temperature balance.

Carbon dioxide, methane, nitrous oxide, hydroflurocarbons (HFC), chlorofluorocarbons (CFC), and perfluorocarbons (PFC) are the most common anthropogenic greenhouse gases. Carbon dioxide gets wide attention due to its rapidly increasing concentration in the atmosphere. Methane, although a trace gas which having a relatively short average lifetime of 9 years in the atmosphere, is a much more potent greenhouse gas compared to $\mathrm{CO}_{2}$. The concentration of $\mathrm{CH}_{4}$ in the atmosphere has been on the rise at an average rate of $8.9 \mathrm{ppbv} /$ year for the last two decades. ${ }^{1}$ Nearly $45 \%$ of the methane released into the atmosphere is done so by anthropogenic activities. The rest comes from natural sources such as wasteland decomposition, termites, agriculture, and domestic activities. With increasing global temperatures, the methane release rate is expected to increase causing a positive feedback loop for global warming. When all effects are included, it is estimated that $0.9 \mathrm{Wm}^{-2}$ radiative forcing comes from the methane, which is more than half that for $\mathrm{CO}_{2}{ }^{2}$ Likewise, another naturally occurring greenhouse gas, nitrous oxide is about 400 times more potent compared to $\mathrm{CO}_{2} . \mathrm{N}_{2} \mathrm{O}$ is

produced during the burning of fossil fuels and is also released by the soil. ${ }^{3}$ Furthermore, 
in a recent study by Shine and Sturges it is estimated that $40 \%$ of the heat trapped by anthropogenic greenhouse gases in the Earth's atmosphere is due to gases other than $\mathrm{CO}_{2}{ }^{4}$

Other potent greenhouse gases, such as hydrofluorocarbons (HFCs), chlorofluorocarbons (CFCs) and perfluorocarbons (PFCs), are heavily used in the electronics, air conditioning, appliances and carpet manufacturing industries. Production and industrial use of CFCs have been discontinued since it was discovered that they contribute to the destruction of the ozone layer. However, PFCs and HFCs are continually utilized in various industries. In addition to the above uses, PFCs are used as a blood substitute in biological cell cultures and in blood transfusions. ${ }^{5}$ PFCs are also widely used as tracers in atmospheric studies, in tracking leaks in gas lines and electrical transmission lines, ${ }^{6}$ and in tracking carbon sequestration processes. ${ }^{7}$ Although the current concentration of some of these tracer gases have been found to be very small compared to that of $\mathrm{CO}_{2}$, their concentration is on the rise for as long as the data exists. ${ }^{8}$ More importantly, PFCs and HFCs are extremely efficient greenhouse gases as they absorb in the atmospheric infrared window and in some cases have atmospheric lifetimes estimated at thousands of years. ${ }^{9}$ This means that some PFCs and HFCs display the characteristics to impact global temperatures significantly more than $\mathrm{CO}_{2}$ in terms of both short term and long term effects. Although it is estimated that they contribute little to the total radiative forcing at present, with the current rate of increase they will be significant contributors in the future according to some models. ${ }^{4}$ Some hydrofluoroethers have been suggested as substitutes for CFCs and HFCs as carrier compounds for lubricants and for use in the refrigeration industry. Although the hydrofluoroethers have slightly lower 
atmospheric lifetimes due to reactions with the $\mathrm{OH}$ radical and other atmospheric scrubbing agents, they have relatively large instantaneous radiative forcing. ${ }^{10}$

An index was introduced some years ago to quantify the potency of an individual molecule regarding global warming. ${ }^{11}$ This index, the 'Global warming potential' (GWP), is defined as the time integrated radiative forcing of one gas with respect to a reference gas over a time horizon. The absolute GWP depends on the time horizon for which the GWP is considered. However it is pointed out in chapter 6 of the Intergovernmental Panel on Climate Change (IPCC) working group's 2001 report $^{9}$ that the GWP of a molecular species is relatively insensitive to the choice of the time horizon when the atmospheric lifetime of the gas is not substantially different from the response time of the reference gas (for $\mathrm{CO}_{2}$, this is about 150 years ${ }^{9}$ ). The GWP of different molecules can vary significantly. For example over a 20 year time horizon the GWP of $\mathrm{CH}_{4}$ is 72 times and $\mathrm{N}_{2} \mathrm{O}$ is 289 times the GWP of $\mathrm{CO}_{2},{ }^{12}$ while the GWP of CFC-11 $\left(\mathrm{CCl}_{3} \mathrm{~F}\right)$ is 6730 times that of $\mathrm{CO}_{2}$. It is clear GWP is a quantity that is very specific to a molecular species, and that while atmospheric lifetime is a factor, a molecule's radiative forcing is often the deciding factor for large variations in GWPs.

The relative radiative forcing parameter is determined on either a molecule-per mole basis or a kilogram-per-kilogram basis compared to a reference, usually $\mathrm{CO}_{2}{ }^{12}$ Generally, a radiative transfer model is used to determine the radiative forcing for perturbations of greenhouse gases. In these computations a certain concentration of a perturbing gas along with the molecule's infrared profile (absorption bands and strengths) is added to the model. From a molecular standpoint the radiative forcing depends on the total absorption of electromagnetic radiation by a molecule, especially within the so- 
called atmospheric window. While it is established that the radiative forcing can vary significantly for different molecular species, the underlying chemical or physical causes for this variation have not been studied. That is, the GWP of different greenhouse gases measured over the same time horizon can vary significantly due to their inherent chemical natures. The purpose of the present research is to identify the underlying molecular properties that cause GWPs to vary so much amongst different types of molecules. If one is to minimize the impact of anthropogenic materials on global climate change, it is necessary to design better materials that have minimal absorption capabilities in the atmospheric window or shorter atmospheric lifetimes. A number of

studies have addressed how to minimize the atmospheric lifetime of materials, ${ }^{13,14}$ however no studies have addressed how to minimize the absorption capabilities of molecular species in the atmospheric window. In the present report, we present new insights into how properties of a molecule influence the molecular absorption in the atmospheric window and the underlying molecular cause of global warming. Theoretical methods used in the present study are detailed in the next section, followed by Results and Discussion, and then Conclusions in the final section.

\section{Theoretical methods}

The determination of equilibrium structures for all molecules was performed using second order Moller-Plesset perturbation theory (MP2) in conjunction with a double zeta plus polarization and diffuse function basis set denoted DZP++ (indicating 
that diffuse functions are included on hydrogen atoms). ${ }^{15-18}$ All $a b$ initio electronic structure calculations were performed with the Q-Chem3.1 ${ }^{19}$ quantum chemistry program. Harmonic vibrational frequencies and infrared (IR) intensities were computed using the same level of theory. The IR vibrational intensities were computed using the double harmonic approximation (i.e., mechanical and electrical harmonicity). Under the double harmonic approximation, infrared vibrational intensities are proportional to the square of the dipole derivatives. ${ }^{20}$ The Cartesian dipole derivatives obtained at the $\mathrm{MP} 2 / \mathrm{DZP}++$ level of theory have been transformed into the simple internal coordinates for each molecule in order to assess various molecular properties. For simplicity, the simple internal coordinates used are limited to the bond distances and angles of the molecules concerned. Intder2005, ${ }^{21}$ a code for vibrational analysis and nonlinear transformations of quartic force fields (including property force fields) has been used to transform the Cartesian dipole derivatives into simple internal coordinate derivatives.

\section{Results and Discussion}

Although carbon dioxide, methane and nitrous oxide are considered to be the main greenhouse gases, as discussed in the Introduction, there are others, especially the halocarbons. One interesting question then is what are the common dominating factors or collection of factors that make certain molecules effective greenhouse gases and others ineffective? Our initial investigation led to the first observation: certain bond stretch vibrational modes are ideally suited for occurring in the atmospheric IR window region (for purposes of this study, we have defined the atmospheric IR window to be 800 to $1400 \mathrm{~cm}^{-1}$ ). These modes include C-F, C-Cl, C-Br, S-F, N-F vibrational stretches among other vibrational modes. Some bond angle bending and torsions fall within the 
atmospheric window and contribute to global warming albeit in a significantly lesser way for reasons that will be discussed below.

To investigate further, collected in Table 1 are the summed IR intensities of several GHGs plus some other atmospheric trace molecules of interest. Also included is the summed IR intensity for bands that occur in the atmospheric window, and the percentage of the total IR intensity that contributes to the bands occurring in the atmospheric window. The atmospheric lifetime and the GWPs (for a time horizon of 100 years) are taken from Ref. 9. $\quad$ It is evident that some of the most effective GHGs have GWPs that are thousands of times that of $\mathrm{CO}_{2}$. In fact, $\mathrm{CFCs}$, which fall into this category, have been recommended for use in the Martian atmosphere to keep Mars warm and habitable. ${ }^{22}$ A systematic inspection into the IR vibrational frequencies and intensities of some of the most common HFCs and CFCs listed in Table 1 has led to following conclusions: 1) the IR absorption intensities within the IR atmospheric window are much larger than that of $\mathrm{CO}_{2}$ and therefore they are much more potent absorption agents than $\mathrm{CO}_{2}$; and 2) the percentage of the integrated vibrational absorption intensity that falls within the atmospheric window increases, up to as high as $99 \%$ of the total IR intensity, as more and more halogens are involved. These factors, boosted by very long atmospheric lifetimes contribute to making the HFCs, CFCs, PFCs, and other GHGs extremely potent compared to $\mathrm{CO}_{2}$ on a per molecule basis. The data reported in Table 1 highlights that it is not only the fact that these molecules posses long atmospheric lifetimes, but even more important is their inherent capability to strongly absorb radiation in the atmospheric window that make them worthy of attention. The HFCs, CFCs, PFCs, and sulfur and nitrogen fluorides not only absorb in the atmospheric window, where no 
other atmospheric molecules absorb, but do so very effectively. The fact that for many of these molecules more than $85-90 \%$ of their IR absorption occurs in the atmospheric window was perhaps not well understood or not well appreciated.

Comparison of the total IR intensity occurring within the atmospheric window for similar $\mathrm{F}$ and $\mathrm{Cl}$ containing molecules contained in Table 1 (e.g., $\mathrm{CF}_{4}$ vs. $\mathrm{CCl}_{4}$ or $\mathrm{CHF}_{3}$ vs. $\mathrm{CHCl}_{3}$ ) shows that $\mathrm{F}$ containing molecules are much more effective greenhouse gases compared to $\mathrm{Cl}$ containing molecules. In addition, we note that the molecule with the largest total IR intensity in the atmospheric window is $\mathrm{SF}_{5} \mathrm{CF}_{3}(1823 \mathrm{Km} / \mathrm{mol})$, and while $\mathrm{SF}_{6}$ has a total IR intensity of $434 \mathrm{Km} / \mathrm{mol}$ in the atmospheric window, it is much smaller in comparison. Of the molecules included in Table 1, only three molecules have a total IR intensity in the atmospheric window greater than $1000 \mathrm{Km} / \mathrm{mol}-\mathrm{CF}_{4}(1403), \mathrm{CClF}_{3}$ (1199), and $\mathrm{SF}_{5} \mathrm{CF}_{3}$ (1823), but considering that the total IR intensity in the atmospheric window for $\mathrm{CO}_{2}, \mathrm{CH}_{4}$, and $\mathrm{N}_{2} \mathrm{O}$ is only $25.7,44.1$, and $13.6 \mathrm{Km} / \mathrm{mol}$, respectively, even the compounds with total IR intensity in the atmospheric window in the hundreds of $\mathrm{Km} / \mathrm{mol}$ are significant greenhouse gases.

A systematic study of the HFCs and PFCs, see Table 2, reveal that with introduction of each $\mathrm{F}$ atom, more and more vibrational modes occur in the atmospheric window by virtue of the C-F bond stretch falling within the $800-1400 \mathrm{~cm}^{-1}$ region, while simultaneously each C-F stretch is becoming more intense. Figure 1 shows graphically that as one introduces $\mathrm{F}$, going from $\mathrm{CH}_{4}$ to $\mathrm{CF}_{4}$, the percentage of the total amount of IR intensity within the atmospheric window increases from $20 \%$ up to almost $100 \%$. It is important to emphasize that simultaneously the absolute IR intensity of the C-F stretches 
increases along this series. For example, the IR intensity per C-F bond in the $\mathrm{CH}_{3} \mathrm{~F}$ to $\mathrm{CF}_{4}$ series is $117,193.5,253.7$, and $350.8 \mathrm{Km} / \mathrm{mol}$, respectively (see Table 2).

Under the double harmonic approximation a vibrational band IR intensity is proportional to the square of the dipole derivative. Therefore a large intensity is the manifestation of a larger dipole derivative. The dipole derivatives calculated for some GHGs in the simple internal coordinate system are presented in Table 3. For a systematic test of the above we computed the dipole derivatives of the C-H, C-F, C-Cl, and N-F bonds for a series of molecules in the simple internal coordinate system. For the $\mathrm{CH}_{4}$, $\mathrm{CH}_{3} \mathrm{~F}, \mathrm{CH}_{2} \mathrm{~F}_{2}, \mathrm{CHF}_{3}$ and $\mathrm{CF}_{4}$ series the individual dipole derivatives increase at first and then decrease slightly. However, the integrated dipole derivative increases linearly as seen in Figure 2a. On going from $\mathrm{CH}_{4}$ to $\mathrm{CH}_{3} \mathrm{~F}$ to $\mathrm{CH}_{2} \mathrm{~F}_{2}$ the dipole derivative increases due to a greater electronegativity difference between $\mathrm{C} / \mathrm{F}$ compared to $\mathrm{C} / \mathrm{H}$. However, the dipole derivative does not increase as one might have expected, and in fact it decreases on going from $\mathrm{CH}_{2} \mathrm{~F}_{2}$ to $\mathrm{CHF}_{3}$ to $\mathrm{CF}_{4}$. This can be rationalized by looking at the Figure 3. Each additional $\mathrm{F}$ renders the central $\mathrm{C}$ more positive. As the ionic character or the oxidation state of the central $\mathrm{C}$ increases so does its electron attracting ability, i.e. electronegativity. Therefore as the central $\mathrm{C}$ becomes more cationic in nature its absolute electronegativity increases and the electronegativity difference between it and the terminal fluorines decreases. The smaller electronegativity difference yields smaller dipole derivatives. However, the integrated dipole derivatives for the C-F stretching modes increases across the entire series as seen in Figure 2a. Likewise the dipole derivatives increase on going from $\mathrm{NH}_{3}$ to $\mathrm{NH}_{2} \mathrm{~F}$ to $\mathrm{NHF}_{2}$ to $\mathrm{NF}_{3}$ (see Figure $2 \mathrm{~b}$ ). Figures $2 \mathrm{~b}$ and $2 \mathrm{~d}$ show that the integrated IR intensities increase even more rapidly 
across both series due to the fact that they are proportional to the square of the dipole derivatives.

Fluorine, being strongly electronegative, forms very polar bonds with the central atom whether that be $\mathrm{C}$ or $\mathrm{N}$. Each additional $\mathrm{F}$ atom makes the central atom more positively charged by drawing electronic charge away, making all of the C-F or N-F bonds more and more polar across the series. We have attempted to quantify this effect by computing a "bond dipole" using either Mulliken populations ${ }^{23}$ or the Natural Population Analysis (NPA). ${ }^{24}$ These are collected in Table 3 together with the percent IR intensity in the atmospheric window from Table 1, for comparison. We note that the electrostatic interaction between the central atom, which is positively charged, and the negatively charged terminal fluorine also causes gradual bond shortening. The bond shortening acts as a mitigating factor for the bond dipole. Hence, although electronegativity difference between the central and the terminal atoms qualitatively decide the ionic nature of the bond, it is hardly the only contributing factor. ${ }^{25}$ Mulliken qualitatively broke down the dipole moment of a bond into four components; primary, overlap, hybridization and core. ${ }^{26}$ The primary moment originates due to electronegativity difference between atoms. While the electronegativity difference is one of the contributing factors to the overall asymmetry in the electronic cloud of the bond, other factors can also contribute and are sometimes dominant. Therefore an attribution of a trend in the dipole derivatives or even the bond dipoles to merely electronegativity differences of the atoms involved would be an oversimplification. Nevertheless, electronegativity differences play a vital role in creating asymmetry in the electronic environment of a bond, and in the case of the X-F bonds studied here is the dominant 
contribution to the bond dipole. To summarize, the highly polarized nature of the X-F bond leads to a large dipole derivative; increasing the number of fluorine atoms bonded to a given central atom increases the total or integrated dipole derivative linearly as a function of the number of $\mathrm{F}$ atoms, leading to a non-linear increase in IR intensity. Therefore a combination of factors, 1) the X-F stretching frequencies falling within the atmospheric IR window; 2) the strong electronegativity of F; and 3) the typically long atmospheric lifetimes of compounds containing many fluorine atoms (since they do not usually readily react with $\mathrm{OH}$ ) contribute towards making fluorine containing compounds the most effective global warming agents.

\section{Conclusions}

As a rule of thumb, a large atomic electronegativity leads to polar bonds by drawing charge from its bonding partner. The total bond polarity or the bond dipole moment increases as the electronegativity difference increases, and a larger bond dipole yields a larger bond dipole derivative leading to a large IR intensity. The total or integrated IR intensity for a given molecule, however, depends on the number of polar bonds, as well as the polarity of each bond. Therefore, since fluorine is highly electronegative, molecules containing several fluorine atoms are strong greenhouse gases and are much more effective warming agents compared to equivalent $\mathrm{Cl}$ and/or $\mathrm{H}$ containing species. With the addition of each $\mathrm{F}$, the integrated bond dipole derivative for the molecule increases linearly and hence the total IR absorption intensity increases dramatically. Moreover, the increased IR absorption contributes to a molecule's radiative 
forcing since the C-F stretching frequencies occur in the atmospheric window. In summary, large dipole derivatives for the vibrational modes that fall within the atmospheric IR window yield very large integrated IR intensities for the HFCs, PFCs and CFCs - sometimes an order of magnitude more than that of the common greenhouse gasses like $\mathrm{CO}_{2}, \mathrm{O}_{3}, \mathrm{~N}_{2} \mathrm{O}$ and $\mathrm{CH}_{4}$. The insights developed in this study should help in better understanding the physical characteristics of greenhouse gases, and specifically what makes an efficient greenhouse gas on a molecular level. It is hoped that the results from this study will be used in the design of more environmentally friendly materials.

\section{Acknowledgements}

PPB gratefully acknowledges support from an appointment to the NASA postdoctoral program at the Ames Research Center, administered by Oak Ridge Associated Universities through a contract with NASA. TJL would like to thank Professor Russell Pitzer for his considerable and thoughtful help during the early stages of his career. 
Table 1. Atmospheric lifetimes, Global Warming Potentials (GWP), Integrated infrared absorption intensities, integrated infrared intensities in the atmospheric IR window and the per cent IR intensity in the Atmospheric window for some common greenhouse gases.

\begin{tabular}{|c|c|c|c|c|c|c|}
\hline Industrial Name & $\begin{array}{l}\text { Chemical } \\
\text { name }\end{array}$ & $\begin{array}{l}\text { Lifetime } \\
\text { Yrs }\end{array}$ & $\begin{array}{l}\text { GWP } \\
100 \mathrm{yr}\end{array}$ & $\begin{array}{l}\text { Int. IR } \\
\text { Intensity } \\
\mathrm{Km} / \mathrm{mol}\end{array}$ & $\begin{array}{l}\text { Intensity in } \\
\text { Atmospheric } \\
\text { window } \\
\mathrm{Km} / \mathrm{mol}\end{array}$ & $\%$ \\
\hline Water & $\mathrm{H}_{2} \mathrm{O}$ & & & 159.0 & 0.0 & 0.0 \\
\hline Carbon dioxide & $\mathrm{CO}_{2}$ & $100^{\mathrm{a}}$ & 1 & 594.5 & 25.7 & 4.1 \\
\hline Nitrous oxide & $\mathrm{N}_{2} \mathrm{O}$ & 114 & 298 & 351.3 & 13.6 & 3.88 \\
\hline Methane & $\mathrm{CH}_{4}$ & 12 & 25 & 127.5 & 44.1 & 34.6 \\
\hline Methyl fluoride & $\mathrm{CH}_{3} \mathrm{~F}$ & 2.6 & 97 & 241.1 & 119 & 49.4 \\
\hline HFC-32 & $\mathrm{CH}_{2} \mathrm{~F}_{2}$ & 4.9 & 675 & 535.6 & 406.3 & 75.9 \\
\hline HFC-23 & $\mathrm{CHF}_{3}$ & 270 & 14800 & 947.6 & 759.6 & 80.2 \\
\hline PFC-14 & $\mathrm{CF}_{4}$ & 50000 & 7390 & 1419.6 & 1403 & 98.8 \\
\hline CFC-13 & $\mathrm{CClF}_{3}$ & 640 & 14400 & 1225.63 & 1199 & 97.9 \\
\hline CFC-12 & $\mathrm{CCl}_{2} \mathrm{~F}_{2}$ & 100 & 10900 & 979.79 & 970.1 & 99.0 \\
\hline CFC-11 & $\mathrm{CCl}_{3} \mathrm{~F}$ & 45 & 4750 & 707.4 & 705.2 & 99.7 \\
\hline Methyl Chloride & $\mathrm{CH}_{3} \mathrm{Cl}$ & 1 & 13 & 109 & 26 & 23.9 \\
\hline Methylene chloride & $\mathrm{CH}_{2} \mathrm{Cl}_{2}$ & 0.38 & 8.7 & 198.7 & 168.5 & 84.8 \\
\hline Chloroform & $\mathrm{CHCl}_{3}$ & 0.5 & 30 & 333.7 & 327.6 & 98.2 \\
\hline Carbon tetrachloride & $\mathrm{CCl}_{4}$ & 26 & 1400 & 443.73 & 443.7 & 99.9 \\
\hline Methyl bromide & $\mathrm{CH}_{3} \mathrm{Br}$ & 0.7 & 5 & 80.3 & 40.7 & 50.7 \\
\hline HCFC-22 & $\mathrm{CHClF}_{2}$ & 12 & 1810 & 769.3 & 742 & 96.5 \\
\hline Ammonia & $\mathrm{NH}_{3}$ & & & 278.02 & 217 & 78.0 \\
\hline Nitrogen trifluoride & $\mathrm{NF}_{3}$ & 740 & 17200 & 519.9 & 516.2 & 99.3 \\
\hline Sulfur hexafluoride & $\mathrm{SF}_{6}$ & 3200 & 22800 & 554.2 & 434 & 78.3 \\
\hline $\mathrm{SF}_{5} \mathrm{CF}_{3}$ & $\mathrm{SF}_{5} \mathrm{CF}_{3}$ & $>1000$ & 18000 & 2029.5 & 1823 & 89.8 \\
\hline Sulfur dioxide & $\mathrm{SO}_{2}$ & & & 172.36 & 137.1 & 79.5 \\
\hline Phosphine & $\mathrm{PH}_{3}$ & & & 303.92 & 64.77 & 21.3 \\
\hline Trifluorophosphine & $\mathrm{PF}_{3}$ & & & 662.46 & 619.8 & 93.6 \\
\hline Ozone & $\mathrm{O}_{3}$ & & & 1802.2 & 1.69 & 0.1 \\
\hline Oxygendifluoride & $\mathrm{OF}_{2}$ & & & 76.44 & 75.91 & 99.3 \\
\hline
\end{tabular}

